\title{
An Efficient Method for Pivoting Free Variables in Linear Programming: A Computational Approach
}

\author{
Syed Inayatullah, \\ Asma Rani, \\ Tanveer Ahmed Siddiqi, \\ Hina Zaheer, \\ Muhammad Imtiaz, \\ Hafsa Athar Jafree,
}

Department of Mathematics, University of Karachi, Karachi, Pakistan

URL:http://dx.doi.org/10.19044/esj.2019.v15n9p1

\begin{abstract}
Commonly used simplex method to solve linear programming problem do not allow variables to be negative during solution process and suggest to break each free variable (variable allowed to be negative) into difference of two non-negative variables. This transformation significantly increases the number of variables as well as after this the problem leaves its original variable space., thus making the geometry of problem (during solution process) difficult to handle and understand. In this paper, we developed a natural generalization of simplex pivots for free variables. Described approach is capable of handling any general linear programming in its original variable space. In our computational study, the primary results showed that the new method outperforms simplex method on general LPs.
\end{abstract}

Keywords: Linear programming, unrestricted variables, simplex method, decomposition

\section{Introduction}

Since 1947, after World War II, linear programming has gained importance amongst the researchers of different fields (Dantzig, 1963). Today because of its tremendous impact in various disciplines, it has become a core research area of many Mathematicians, Economists and Decision Scientists. Linear programming is the optimization of an outcome based on some set of constraints using a linear mathematical model. It deals with maximizing (minimizing) of a linear function over a convex polyhedron specified by a set of linear constraints. The origin of developing algorithms to solve a given 
system of linear inequalities actually goes back to the $19^{\text {th }}$ century, where they were first studied by Fourier (Grattan-Guinness, 1970). Later, several mathematicians such as Dines (1918) and Motzkin (1952) rediscovered these algorithms. Simplex method developed by Dantzig (1963), which is specially designed to solve LPs with non-negative variables and so far, the most preferred method for solving LPs because of its efficiency (Shamir, 1987).

We categorize the variables in an LP in two kinds, first kind is nonnegative variables, i.e. the variables having explicit non-negativity restrictions and the second kind is free/unrestricted variables, i.e. variables having no explicit non-negativity restriction.

Just a few versions of simplex algorithm presented in the literature and textbooks for solving LPs with free variables, which mostly initiated by decomposing the free variables as a difference of two non-negative variables thus converting it into an LP with explicit non-negativity restrictions on all variables. Dantzig stated in (Dantzig, 1963) another decomposition technique, which requires insertion of a single additional variable to the problem, and attributed this decomposition to A. W. Tucker. Later on, Schechter (1991) has presented geometrical interpretation of above technique, but in 1985, Gass (1985) had already proven that defining free variables as difference of two nonnegative variables is computationally inefficient.

For larger LPs, implementation of the simplex method with the decomposition of free variables increases the number of variables and importantly loses the geometry of problem in the original variable space. Furthermore, this makes a technically incorrect impression on the reader that linear programming with free variables is perhaps a special case of linear programming with non-negative variables. Actually linear programming with unrestricted variables is a generalization of linear programming with nonnegative variables, so there must be a generalized way of choosing entering and leaving basic variables that can directly deal unrestricted variables and as well as non-negative variables.

Orchard-Hays (1968), Spivey and Thrall (1970), Gass (1985) and Dantzig and Thapa (1997) discussed a way of handling free variables in terms of explicit representation within a simplex tableau format. But that method lacks reliability from the perspective of efficiency on large LPs, because of the randomness involved in the selection of initial explicit representations.

Here, this paper would reveal a similar but systematic and efficient procedure that could directly handle unrestricted variables in solving general LPs. 


\section{A Linear Programming Problem}

A general LP problem with mix kind of variables could be defined as,

$$
\begin{array}{cc}
\text { Maximize } & z=\mathbf{c}^{T} \mathbf{x} \\
\text { subject to } & A \mathbf{x}=\mathbf{b} \\
\text { where } & \mathbf{x}=\left[\begin{array}{l}
\mathbf{x}_{U} \\
\mathbf{x}_{O}
\end{array}\right], \mathbf{x}_{O} \geq 0, \mathbf{x} \in \mathfrak{R}^{n}
\end{array}
$$

where, $U$ as index set of variables that have no explicit bound, and $O$ as index set of variables that have explicit non-negativity conditions, $A \in \mathfrak{R}^{m \times n}, \mathbf{b} \in \mathfrak{R}^{m}, \mathbf{c} \in \mathfrak{R}^{n}$ and $m \leq n$. It is assumed that $A$ is full rank.

Let $B$ be the set of indices of the variables in the basis, and $N$ be the set of indices of variables in the non-basis, such that $A_{B}$ is invertible, and nonbasis $N:=\{1, \cdots, n\} \backslash B$. We may write

$$
\begin{aligned}
& A_{B} \mathbf{x}_{B}+A_{N} \mathbf{x}_{N}=\mathbf{b} \\
\Rightarrow & \mathbf{x}_{B}+A_{B}^{-1} A_{N} \mathbf{x}_{N}=A_{B}^{-1} \mathbf{b}
\end{aligned}
$$

Now by substituting the value of $\mathbf{x}_{B}$ from equation (2), the objective of system (1) can be reformulated as

$$
\begin{aligned}
& z-\left(\mathbf{c}_{B}^{T} \mathbf{x}_{B}+\mathbf{c}_{N}^{T} \mathbf{x}_{N}\right)=0 \\
\Rightarrow & z-\mathbf{c}_{B}^{T}\left(A_{B}^{-1} \mathbf{b}-A_{B}^{-1} A_{N} \mathbf{x}_{N}\right)-\mathbf{c}_{N}^{T} \mathbf{x}_{N}=0 \\
\Rightarrow & z=\left(\mathbf{c}_{N}^{T}-\mathbf{c}_{B}^{T} A_{B}^{-1} A_{N}\right) \mathbf{x}_{N}+\mathbf{c}_{B}^{T} A_{B}^{-1} \mathbf{b}
\end{aligned}
$$

The following collection of equations along with non-negativity condition on variables $\mathbf{x}_{0}$,

$$
\frac{\mathbf{x}_{B}+A_{B}^{-1} A_{N} \mathbf{x}_{N}=A_{B}^{-1} \mathbf{b}}{\operatorname{Max} z=\left(\mathbf{c}_{N}^{T}-\mathbf{c}_{B}^{T} A_{B}^{-1} A_{N}\right) \mathbf{x}_{N}+\mathbf{c}_{B}^{T} A_{B}^{-1} \mathbf{b}}
$$

are termed as dictionary of the LP system (1) for basis $B$.

The dictionary data, for any basis $B$, may be elementwise represented in the following collection of equations, denoted by $D(B)$, which is slightly modified form of (Chvatal, 1983) (Kaluzny, 2001).

$$
D(B)=\left\{\begin{array}{c}
x_{i}+\sum_{j \in N} \alpha_{i j} x_{j}=\beta_{i}, i \in B \\
\text { Maximize } z=\sum_{j \in N} \gamma_{j} x_{j}+\hat{z}
\end{array}\right\}
$$


Where $\beta_{i}$ is the component of vector $A_{B}^{-1} \mathbf{b} \in \mathfrak{R}^{B}$ representing value of the basic variable $x_{i}, \quad \alpha_{i j}$ is the element of $A_{B}^{-1} A_{N} \in \mathfrak{R}^{B \times N}$ denoting the coefficient of the non-basic variable $x_{j}$ in the equation containing basic variable $x_{i}, \gamma_{j}$ is the component of $\left(\mathbf{c}_{N}^{T}-\mathbf{c}_{B}^{T} A_{B}^{-1} A_{N}\right)^{T} \in \mathfrak{R}^{N}$ representing the coefficient of non-basic variable $x_{j}$ in the objective function of the current dictionary, and $\hat{z}=\mathbf{c}_{B}^{T} A_{B}^{-1} \mathbf{b} \in \mathfrak{R}$ is the objective scalar value associated with current basis $B$. A basis $B$ (or a dictionary $D(B)$ ) is said to be feasible if $\beta_{i} \geq 0$ for all $i \in O$.

\section{Selection of entering and leaving variables:}

The foremost requirement in solving a linear programming problem by simplex method is an initial basic feasible solution. Geometrically, it lies at the origin. The simplex method then iterates along edges to adjacent corner points of the feasible region in search of a better objective value. Algebraically, step of the selection of moving edge is known as the selection of entering basic variable.

Consider the following LP,

$$
\begin{array}{ll}
\begin{array}{l}
\text { Maximize } Z=10 x_{1}-100 x_{2}+9 x_{3}-110 x_{4} \\
\text { subject to }
\end{array} & \\
\begin{array}{ccc}
2 x_{1}+3 x_{2}+3 x_{3}-2 x_{4}+x_{5} & =12 \\
x_{1}-x_{2}-5 x_{3}+x_{4}+x_{6} & =20 \\
-3 x_{1}+4 x_{2}+8 x_{3}+4 x_{4} & +x_{7} & =24 \\
2 x_{1}-4 x_{2}-5 x_{3}-6 x_{4} & +x_{8} & =60 \\
3 x_{1}-4 x_{2}-3 x_{3}-2 x_{4} & +x_{9} & =12
\end{array} \\
x_{3}, x_{4}, x_{5}, x_{6}, x_{7}, x_{8} \geq 0 &
\end{array}
$$

Here $x_{1}, x_{2}$ and $x_{9}$ could be treated as unrestricted variables because there are no bounds on their values mentioned explicitly, set $U=\{1,2,9\}$. Whereas $x_{3}$, $x_{4}, x_{5}, x_{6}, x_{7}$ and $x_{8}$ are termed as non-negative variables because of an explicit description of zeros as their lower bounds, set $O=\{3,4,5,6,7,8\}$.

For the initial feasible basis $B$, setting $B=\{5,6,7,8,9\}$, is the easiest choice. Therefore corresponding $N=\{1,2,3,4\}$, the initial solution $(0,0,0,0,12,20,24,60,12)$ with $Z=0$ is obtainable by plugging in the values of 
non-basic variables equal to zero. From the expression of objective function it is clear that any increase in values of the non-basic variables $x_{1}$ and $x_{3}$ have positive impact and $x_{2}$ and $x_{4}$ have negative impact on objective value $Z$. In other words, one could say that to increase the value of $Z$ the non-basic $x_{1}$ and $x_{3}$ could be increased while $x_{2}$ and $x_{4}$ could instead be decreased. But $x_{4}$ already on its non-negativity lower bound so could not be decreased any more. In short, $x_{1}, x_{2}$ and $x_{3}$ are candidate entering variables of which $x_{1}$ and $x_{3}$ are candidate increasing and $x_{2}$ is candidate decreasing (here $x_{4}$ is not considered as candidate decreasing variable).

The rules of selecting an entering basic variable among all candidate entering variables are usually known as Pricing Rules. So far many pricing rules have been developed for non-negative variables, some of which are, Dantzig's largest coefficient rule (Dantzig, 1963), steepest edge rule (1977), Devex rule (Harris, 1973), Minimum angle method (Inayatullah, Khan, Imtiaz, $\&$ Khan, 2010), Largest-distance rule (Pan, 2008), Nested Pricing rule (Pan, 2008) Nested largest-distance rule (Pan, 2010). Here in this paper we are using a generalization of Dantzig's largest coefficient method. According to this criterion, most preferred entering variable is the variable along which $Z$ has highest increasing rate. In contrast to Dantzig's original method here entering variable would not be necessarily increasing and may be decreasing as well. In the example defined above $x_{2}$ is a preferred entering variable, because one unit decrease in its value results a 100 units increase in the value of $Z$, which is highest with respect to other candidate variables.

For any increasing (decreasing) variable $x_{i}$, a variable $x_{j}$ is said to be leaving variable if $x_{j}$ provides most stringent bound on the increase (decrease) of variable $x_{i}$.

As in the example defined above,

- if $x_{1}$ is entering variable then $x_{5}$ would be the leaving variable, because as $x_{1}$ increased to value $6, x_{5}$ get struck with its zero lower bound firstly among other basic variables.

- If $x_{2}$ were selected as entering variable then $x_{8}$ would be the leaving variable, because as $x_{2}$ decreased to $-15, x_{8}$ reached its zero lower bound quicker than other basic variables. 
- If $x_{3}$ were selected as entering variable then $x_{7}$ is the leaving variable because as $x_{3}$ increased to value $3, x_{7}$ get struck with its zero lower bound firstly among other basic variables.

Note: Unrestricted variables would never become a leaving variable because they didn't have any upper or lower bound.

\section{General rule:}

For Dictionary 0, let $R_{1}$ be index sets of increasing variables in $\mathbf{x}_{N}$, Clearly, $R_{1}:=\left\{j: \gamma_{j}>0, j \in N\right\}$. Let $R_{2}$ is the index set of decreasing variables in $\mathbf{x}_{N \cap U}$. Clearly $R_{2}:=\left\{j: \gamma_{j}<0, j \in N \cap U\right\}$. So, index set of preferred entering variables among all the non-basic variables would be defined by, $R=\left\{j: j=\arg \max \left\{\gamma_{R_{1}},-\gamma_{R_{2}}\right\}\right\}$. If $R$ gets only a single element, say $k$, then $x_{k}$ would be preferred entering variable and if $R$ gets multiple elements then choice could be arbitrary.

For leaving variable, since the variables in $\mathbf{x}_{B \cap U}$ has no upper or lower bound, they have no reason to leave the basis. So the leaving variable is chosen from $\mathbf{x}_{B \cap O}$ only, by performing the following ratio test: "If $k \in R_{1}$ then index of the leaving variable is obtained by $r=\arg \min \left\{\beta_{i} / \alpha_{i k}: \alpha_{i k}>0, i \in B \cap O\right\}$, while if $k \in R_{2}$ then $r=\arg \min \left\{-\beta_{i} / \alpha_{i k}: \alpha_{i k}<0, i \in B \cap O\right\} "$

\section{Theorem 1: Optimality condition}

A feasible basis $B$ is said to be optimal if in associated dictionary $\gamma_{j} \leq 0$ $\forall j \in O$ and $\gamma_{j}=0 \forall j \in U$.

Proof:

$\gamma_{j} \leq 0$ for all $j \in O$ implies that there is no non-negative non-basic variable available that could be used to increase the value of $Z$ without violating its zero lower bound, and $\gamma_{j}=0$ for all $j \in U$ implies that there is no free nonbasic variable available. Hence it shows optimality of current basis. 


\section{Theorem 2: Unboundedness condition}

The linear program (1) is unbounded if it has a feasible basis $B$ and in the associated dictionary there exists either $\gamma_{j}>0, j \in N$ such that $\alpha_{B j} \leq 0$ or $\gamma_{j}<0, j \in N \cap U$ such that $\alpha_{B j} \geq 0$.

Proof:

Consider the case of $\gamma_{j}>0, j \in N$ and $\alpha_{B j} \leq 0$ which implies that from current (feasible) basis one can increase the value of $x_{j}$ indefinitely and the objective value will increase in direct proportion to $x_{j}$.

Now consider the case of $\gamma_{j}<0, j \in N \cap U$ and $\alpha_{B j} \geq 0$ which implies that from current (feasible) basis one can decrease the value of $x_{j}$ indefinitely and the objective value will increase in direct proportion to decrease in $x_{j}$.

\section{Description of the procedure:}

\section{Problem}

Given a dictionary $D(B)$, with index set $U$ of free variables and the index set $O$ of nonnegative variables. Obtain an optimal basis.

\section{Algorithm}

Step 1: Let $R_{1} \subseteq N$ such that $R_{1}:=\left\{j: \gamma_{j}>0, j \in N\right\}$,

$R_{2} \subseteq N \cap U$ such that $R_{2}:=\left\{j: \gamma_{j}<0, j \in N \cap U\right\}$

If $R_{1} \cup R_{2}=\phi$ then $D(B)$ is optimal. Exit.

Step 2: Set $R:=\left\{j: j=\arg \max \left\{\gamma_{R_{1}},-\gamma_{R_{2}}\right\}\right\}$. If $R$ gets only a single element, say $k$, then $x_{k}$ would be entering basic variable and if $R$ gets multiple elements then choice could be made on maximum of these.

Step 3: if $k \in R_{1}$,

$$
r=\arg \min \left\{\beta_{i} / \alpha_{i k}: \alpha_{i k}>0, i \in B \bigcap O\right\}
$$

Otherwise,

$$
r=\arg \min \left\{-\beta_{i} / \alpha_{i k}: \alpha_{i k}<0, i \in B \bigcap O\right\}
$$


Step 4: If $r$ does not exist, then the given problem is unbounded. Exit. Otherwise make a pivot on $(r, k)$.

Set $B=(B \bigcup\{k\}) \backslash\{r\}, N=(N \bigcup\{r\}) \backslash\{k\}$ and update $D(B)$. Go to step 1

\section{Example 1:}

Consider the following LP,

$$
\begin{array}{lc}
\text { Maximize } & z=-82 x_{1}-87 x_{2}-9 x_{3} \\
\text { subject to } & \\
& 19 x_{1}-27 x_{2}+4 x_{3} \leq 11 \\
25 x_{1}+42 x_{2}+50 x_{3} \leq 97 \\
-4 x_{1}-34 x_{2}-42 x_{3} \leq 1 \\
-41 x_{1}+33 x_{2}-5 x_{3} \leq 78
\end{array}
$$

On insertion of non-negative slack variables $x_{4}, x_{5}, x_{6}$ and $x_{7}$ problem becomes,

$$
\begin{array}{cc}
\text { Maximize } & z=-82 x_{1}-87 x_{2}-9 x_{3} \\
\text { subject to } & \\
19 x_{1}-27 x_{2}+4 x_{3}+x_{4}=11 \\
25 x_{1}+42 x_{2}+50 x_{3}+x_{5}=97 \\
-4 x_{1}-34 x_{2}-42 x_{3}+x_{6}=1 \\
-41 x_{1}+33 x_{2}-5 x_{3}+x_{7}=78 \\
x_{4} \geq 0, x_{5} \geq 0, x_{6} \geq 0, x_{7} \geq 0
\end{array}
$$

Initial dictionary for basic variables $x_{4}, x_{5}, x_{6}$ and $x_{7}$ will be,

$$
\begin{array}{r}
x_{4}+19 x_{1}-27 x_{2}+4 x_{3}=11 \\
x_{5}+25 x_{1}+42 x_{2}+50 x_{3}=97 \\
x_{6}-4 x_{1}-34 x_{2}-42 x_{3}=1 \\
x_{7}-41 x_{1}+33 x_{2}-5 x_{3}=78 \\
\hline \text { MaxZ }=-82 x_{1}-87 x_{2}-9 x_{3}+0
\end{array}
$$

Here all the non-basic variables are decreasing variables, according to criteria defined above in section $4, x_{2}$ is most preferred choice to enter in to the basis and then $x_{6}$ would leave the basis. 
After performing the change of basis operations, we would get following system,

$$
\begin{array}{r}
x_{4}+\frac{377}{17} x_{1}-\frac{27}{34} x_{6}+\frac{635}{17} x_{3}=\frac{347}{34} \\
x_{5}+\frac{341}{17} x_{1}+\frac{21}{17} x_{6}-\frac{32}{17} x_{3}=\frac{1670}{17} \\
x_{2}+\frac{2}{17} x_{1}-\frac{1}{34} x_{6}+\frac{21}{17} x_{3}=-\frac{1}{34} \\
x_{7}-\frac{763}{17} x_{1}+\frac{33}{34} x_{6}-\frac{778}{17} x_{3}=\frac{2685}{34} \\
\hline \text { Max } Z=-\frac{1220}{17} x_{1}-\frac{87}{34} x_{6}+\frac{1674}{17} x_{3}+\frac{87}{34}
\end{array}
$$

Here $x_{1}$ is decreasing and $x_{3}$ is increasing variable, in which $x_{3}$ is our preferred choice (see section 4). Then leaving variable would be $x_{4}$.

$$
\begin{gathered}
x_{3}+\frac{377}{635} x_{1}-\frac{27}{1270} x_{6}+\frac{17}{635} x_{4}=\frac{347}{1270} \\
x_{5}+\frac{13447}{635} x_{1}+\frac{759}{635} x_{6}+\frac{32}{635} x_{4}=\frac{62706}{635} \\
x_{2}-\frac{391}{635} x_{1}-\frac{2}{635} x_{6}-\frac{21}{635} x_{4}=-\frac{233}{635} \\
x_{7}-\frac{6146}{347} * x_{1}-\frac{3}{1270} x_{6}+\frac{778}{635} x_{4}=\frac{12715}{139} \\
\text { MaxZ }=-\frac{12632}{97} x_{1}-\frac{591}{1270} x_{6}-\frac{1674}{635} x_{4}+\frac{2033}{69}
\end{gathered}
$$

Here $x_{1}$ is only choice for entering variable. Leaving variable is $x_{7}$.

$$
\begin{gathered}
x_{3}+\frac{377}{11247} x_{7}-\frac{80}{3749} x_{6}+\frac{158}{2329} x_{4}=\frac{1633}{489} \\
x_{5}+\frac{599}{501} x_{7}+\frac{2875}{2411} x_{6}+\frac{944}{623} x_{4}=\frac{3538}{17} \\
x_{2}-\frac{17}{489} x_{7}-\frac{1}{326} x_{6}-\frac{37}{489} x_{4}=-\frac{3469}{978} \\
x_{1}-\frac{347}{6146} x_{7}+\frac{1}{7498} x_{6}-\frac{103}{1489} x_{4}=-\frac{2102}{407} \\
\text { MaxZ }=-\frac{2169}{295} x_{7}-\frac{226}{596} x_{6}-\frac{2981}{256} x_{4}+\frac{19675}{28}
\end{gathered}
$$

Since all the variables are in their allowable range (implies feasibility) and there is no one which could be used to increase $Z$ without violating any constraint. Therefore optimality achieved and the optimal solution is $\left(x_{1}, x_{2}, x_{3}\right)=\left(\frac{-2102}{407}, \frac{-3469}{978}, \frac{1633}{489}\right)$ with $z=\frac{19657}{28}$. 


\section{Example 2:}

Now consider another example,

$$
\begin{array}{lc}
\text { Maximize } & z=15 x_{1}+35 x_{2}+9 x_{3} \\
\text { subject to } & \\
& -30 x_{1}+3 x_{2}-10 x_{3} \leq 26 \\
- & 36 x_{1}+4 x_{2}+18 x_{3} \leq 4 \\
20 x_{1}+37 x_{2}+25 x_{3} \leq 75 \\
\\
-40 x_{1}-x_{2}+3 x_{3} \leq 24
\end{array}
$$

Initial Dictionary:

$$
\begin{gathered}
x_{4}+30 x_{1}-3 x_{2}+10 x_{3}=26 \\
x_{5}+36 x_{1}-4 * x_{2}-18 x_{3}=4 \\
x_{6}-20 x_{1}-37 x_{2}-25 x_{3}=75 \\
x_{7}+40 x_{1}+1 x_{2}-3 x_{3}=24 \\
\hline \operatorname{MaxZ}=+15 x_{1}+35 x_{2}+9 x_{3}+0
\end{gathered}
$$

Iteration 1:

$$
\begin{array}{cc}
x_{4} & -3 x_{1}-\frac{3}{4} x_{5}-\frac{47}{2} x_{3}=23 \\
x_{2} & -9 x_{1}+\frac{1}{4} x_{5}+\frac{9}{2} x_{3}=1 \\
x_{6}+353 * x_{1}-\frac{37}{4} x_{5}-\frac{283}{2} x_{3}=38 \\
x_{7} \quad-49 x_{1}+\frac{1}{4} x_{5}+\frac{15}{2} x_{3}=25 \\
\hline \text { MaxZ }= & +330 x_{1}-\frac{35}{4} x_{5}-\frac{332}{2} x_{3}+35
\end{array}
$$

Iteration 2:

$$
\begin{gathered}
x_{4}+\frac{3}{353} x_{6}-\frac{585}{706} x_{5}-\frac{4817}{195} * x_{3}=\frac{8233}{353} \\
x_{2}+\frac{9}{353} x_{6}+\frac{5}{353} x_{5}+\frac{315}{353} x_{3}=\frac{695}{353} \\
x_{1}+\frac{1}{353} x_{6}-\frac{37}{1412} x_{5}-\frac{283}{706} x_{3}=\frac{38}{353} \\
x_{7}+\frac{49}{353} x_{6}-\frac{365}{353} x_{5}-\frac{4286}{353} x_{3}=\frac{3966}{131} \\
\text { Max } Z=-\frac{330}{353} x_{6}-\frac{145}{1412} x_{5}-\frac{24159}{706} x_{3}+\frac{11707}{166}
\end{gathered}
$$

Iteration 3: 


$$
\begin{array}{r}
x_{3}-\frac{3}{8720} x_{6}+\frac{117}{3488} x_{5}-\frac{195}{4817} x_{4}=-\frac{896}{949} \\
x_{2}+\frac{45}{1744} x_{6}-\frac{55}{3488} x_{5}+\frac{63}{1744} x_{4}=\frac{1684}{599} \\
x_{1}+\frac{47}{17440} x_{6}-\frac{89}{6976} x_{5}-\frac{283}{17440} x_{4}=-\frac{296}{1093} \\
x_{7}+\frac{587}{4360} x_{6}-\frac{1093}{1744} x_{5}-\frac{724}{1473} x_{4}=\frac{4684}{249} \\
\hline \text { MaxZ }=-\frac{1525}{1611} x_{6}+\frac{1111}{10632} x_{5}-\frac{658}{475} x_{4}+\frac{20875}{203}
\end{array}
$$

Here $x_{5}$ is entering variable but there is no leaving variable, which is indication of unbounded optimal solution.

\section{Computational Results:}

Following table presents a comparison of average number of iterations of our algorithm (USM) with Danzig's simplex method (SM) (Dantzig, 1963). Using random models suggested by Kaluzny (2001),

$$
\begin{array}{cc}
\text { Maximize } & \sum_{j=1}^{n} c_{j} \mathbf{x}_{j} \\
\text { subject to } & \sum_{j=1}^{n} a_{i j} \mathbf{x}_{j} \leq \mathbf{b}_{i}, \quad i=1,2, \ldots, m \\
\mathbf{x}_{j} \geq 0, \quad j=1,2, \ldots, n
\end{array}
$$

We generated 250 linear programs with the coefficients $c_{j}, b_{i}$ and $a_{i j}$ chosen randomly from the integer interval $[-50,50]$, and used MATLAB to generate the following results. The results depict that on average USM take much lesser number of iterations than SM, especially on higher order problems. 
Table 1: Average number of iterations on random LPs.

\begin{tabular}{|c|c|c|c|}
\hline Order & USM & SM & $\begin{array}{l}\text { Average Number of } \\
\text { Iterations to be saved } \\
\text { in USM (in \%) }\end{array}$ \\
\hline $3 \times 3$ & 1.58566 & 1.79283 & 11.55547 \\
\hline $3 \times 5$ & 1.64542 & 1.73705 & 5.275035 \\
\hline $3 \times 7$ & 1.5259 & 1.58566 & 3.768778 \\
\hline $5 \times 3$ & 2.53386 & 2.80876 & 9.787237 \\
\hline $5 \times 5$ & 2.7012 & 3.17928 & 15.03737 \\
\hline $5 \times 7$ & 2.60159 & 2.79681 & 6.980095 \\
\hline $7 \times 5$ & 3.98008 & 4.75697 & 16.33161 \\
\hline $7 \times 7$ & 3.83665 & 4.36255 & 12.05488 \\
\hline $7 \times 10$ & 3.67331 & 3.98805 & 7.892078 \\
\hline $10 \times 10$ & 5.77689 & 6.77689 & 14.75603 \\
\hline $10 \times 15$ & 5.41434 & 6.0757 & 10.88533 \\
\hline $15 \times 10$ & 9.0757 & 11.988 & 24.29346 \\
\hline $15 \times 15$ & 8.54183 & 10.2032 & 16.28283 \\
\hline $20 \times 20$ & 12.0518 & 14.9841 & 19.56941 \\
\hline $20 \times 30$ & 11.3586 & 13.2789 & 14.46129 \\
\hline $30 \times 20$ & 19.9562 & 29.3426 & 31.98899 \\
\hline $30 \times 30$ & 18.0598 & 22.7888 & 20.75142 \\
\hline $30 \times 40$ & 17.5777 & 21.9681 & 19.98534 \\
\hline $40 \times 40$ & 24.3984 & 33.0279 & 26.12791 \\
\hline $40 \times 50$ & 23.9801 & 31.4143 & 23.66502 \\
\hline $50 \times 50$ & 30.8446 & 42.3347 & 27.14109 \\
\hline $50 \times 70$ & 30.3825 & 40.9482 & 25.8026 \\
\hline $50 \times 100$ & 29.6175 & 39.4701 & 24.96219 \\
\hline $70 \times 50$ & 47.0199 & 76.9044 & 38.85929 \\
\hline $70 \times 70$ & 43.6614 & 64.3984 & 32.20111 \\
\hline $70 \times 100$ & 43.6175 & 60.8008 & 28.26163 \\
\hline $100 \times 70$ & 69.749 & 125.833 & 44.57018 \\
\hline $100 \times 100$ & 64.5498 & 100.251 & 35.61181 \\
\hline $100 \times 200$ & 62.4064 & 91.4223 & 31.73832 \\
\hline $200 \times 100$ & 205.163 & 403.558 & 49.16146 \\
\hline $200 \times 200$ & 133.769 & 250.06 & 46.50524 \\
\hline $200 \times 300$ & 130.661 & 231.869 & 43.64878 \\
\hline $300 \times 200$ & 223.02 & 533.534 & 58.19948 \\
\hline $300 \times 300$ & 204.243 & 418.183 & 51.15942 \\
\hline $300 \times 400$ & 202.279 & 399.147 & 49.32218 \\
\hline $400 \times 300$ & 289.347 & 699.709 & 58.64752 \\
\hline $400 \times 400$ & 275.598 & 611.841 & 54.95594 \\
\hline $400 \times 500$ & 273.084 & 589.139 & 53.64693 \\
\hline $500 \times 400$ & 355.96 & 881.558 & 59.62149 \\
\hline $500 \times 500$ & 348.578 & 823.964 & 57.695 \\
\hline
\end{tabular}

Furthermore, the comparison between USM and SM illustrates by the graphs between "number of elements in coefficient matrix" versus "average number of iterations" plotted below. 


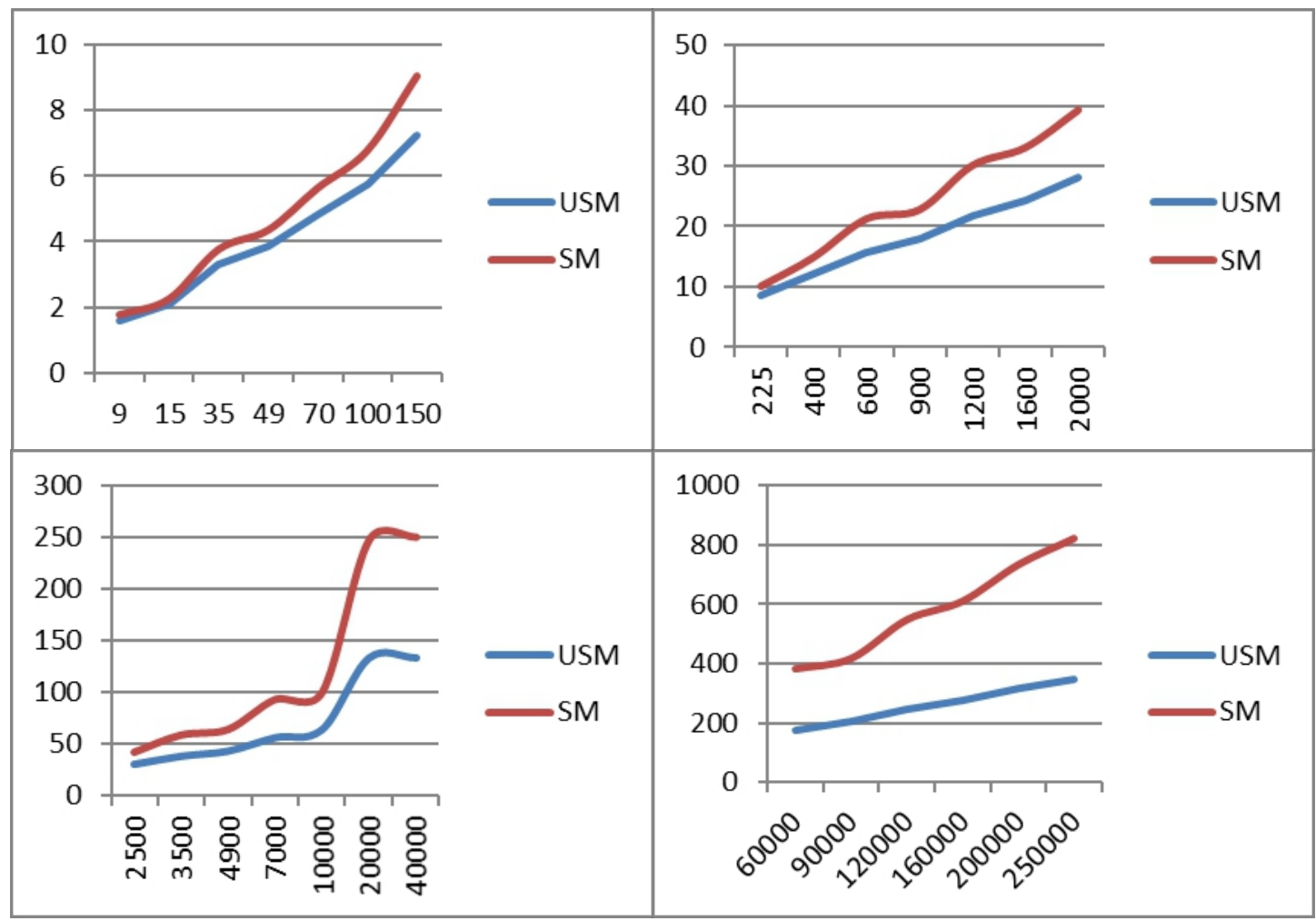

Figure (1): Graphs showing the comparison of average iterations between USM and SM with respect to number of elements in the coefficient matrix. Here numbers of elements in coefficient matrix mentioned on horizontal axis and average number of iterations on vertical axis.

From figure (1), it is clearly observable that USM has greater efficiency for large coefficient matrices. To get a visualization of this increasing trend of efficiency, we also plotted the following graph between relative efficiency of USM and the number of elements in coefficient matrices. 


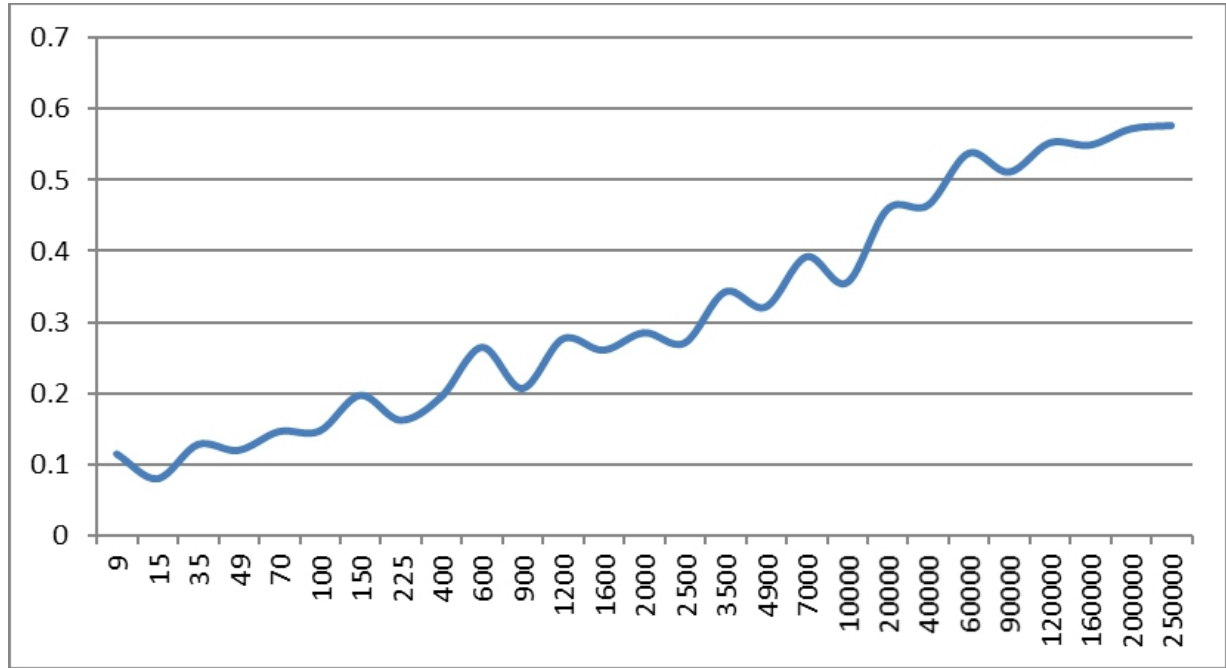

Figure (2): Trend of relative efficiency of USM with respect to number of elements in coefficient matrix. Total number of elements in coefficient matrix mentioned on horizontal axis and fraction of number of average saved iterations mentioned on vertical axis.

Now, to further analyze the trend behavior with respect to order of the coefficient matrices, we observed relationship between $\%$ relative efficiency and the row-column ratio $\left(\frac{m}{n}\right)$ of coefficient matrices, for $m=40,60,80$, and 100.

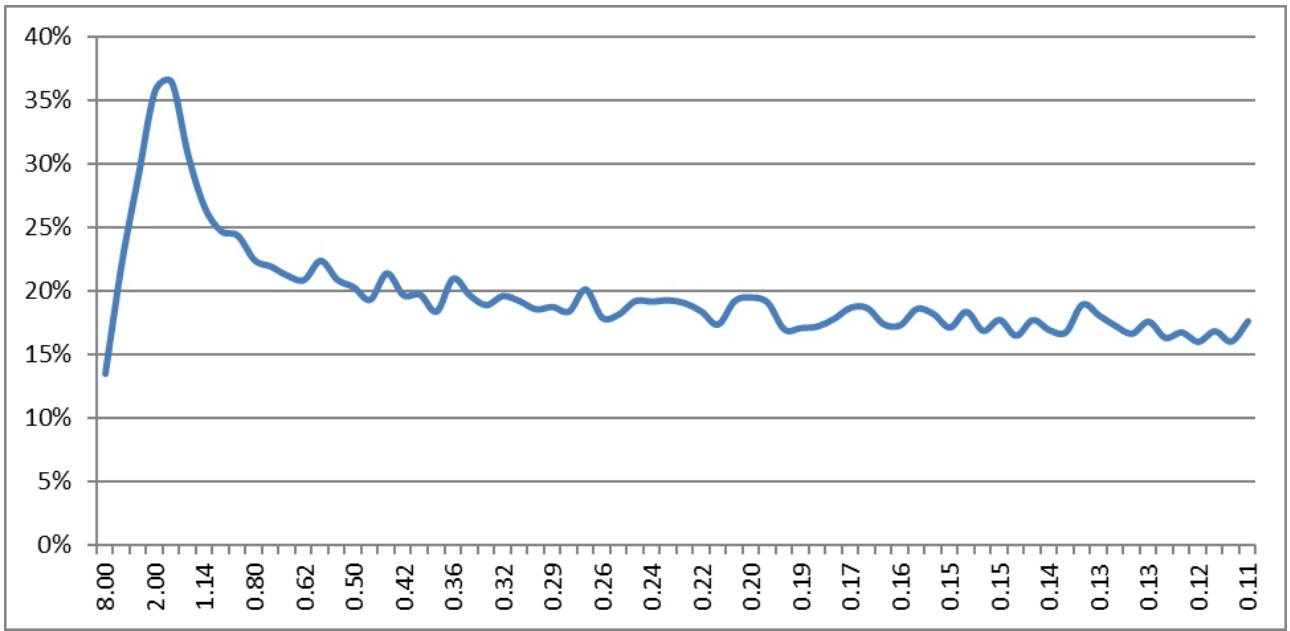

Figure (3): The data obtained by taking $m=40$ and $n=\{5 p \mid p \in[1,70], p \in Z\}$. 


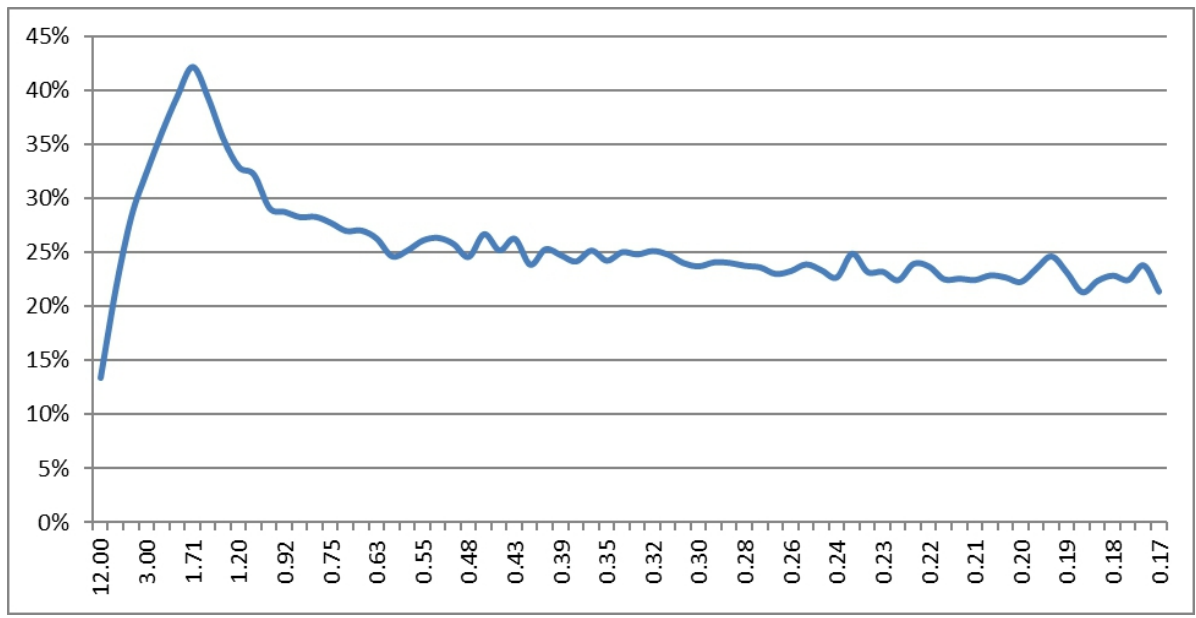

Figure (4): The data obtained by taking $m=60$ and $n=\{5 p \mid p \in[1,70], p \in Z\}$.

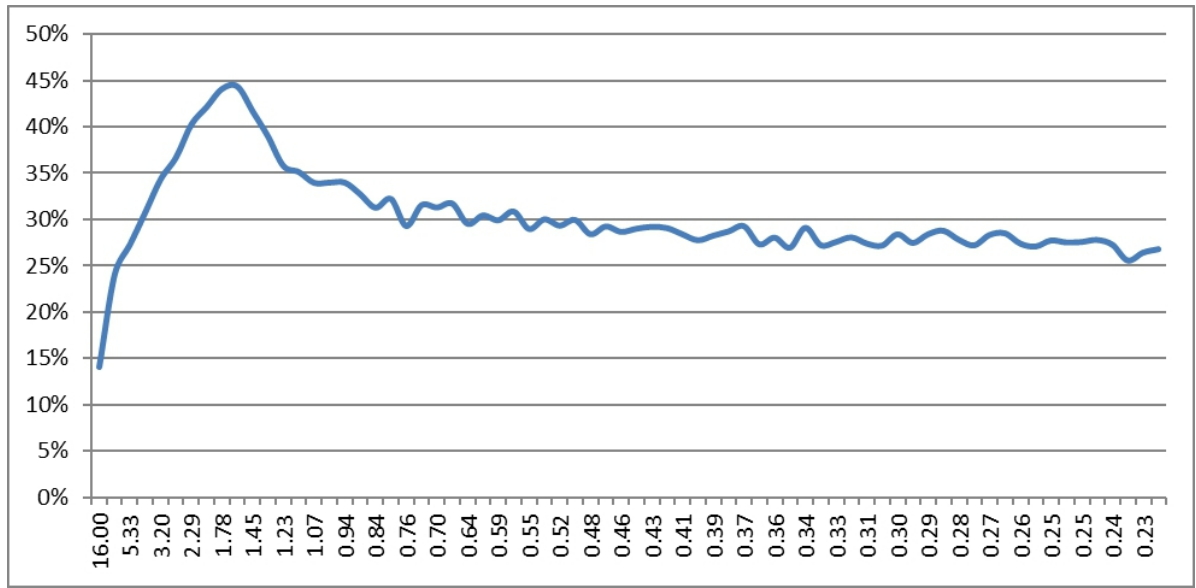

Figure (5): The data obtained by taking $m=80$ and $n=\{5 p \mid p \in[1,70], p \in Z\}$.

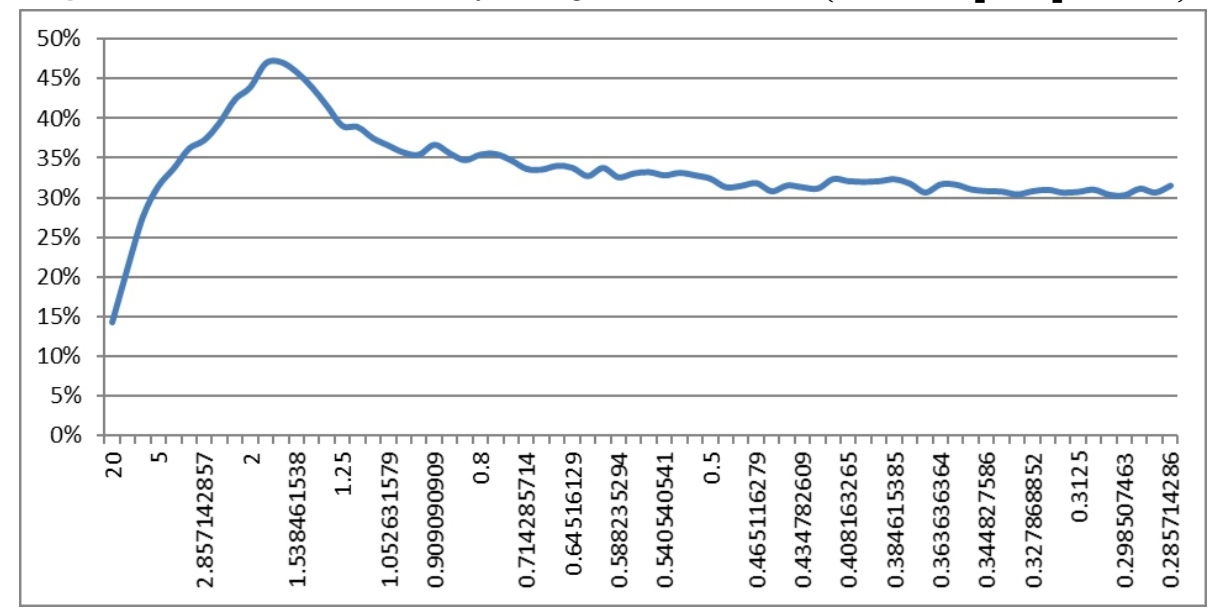

Figure (6): The data obtained by taking $m=100$ with $n=\{5 p \mid p \in[1,70], p \in Z\}$. 
One can notice in figures (3) to (6), as the value of $m$ increases, the relative efficiency of USM also increases, and gradually attain a maximum value when number of constraints are nearly double the number of variables ( $m \approx 2 n$ ), afterwards the trend is approaching to a limiting value for a long run.

\section{Applications}

Although every LP with explicit non-negativity conditions on variables could also be considered as a special case of LP with free variables, besides free variables exclusively arise in a wide number of practical situations too, e.g. production smoothing applications in which decision variables are defined to include periodical differences in production levels which could be positive or negative (Gass, 1985). A linear-programming formulations of zero-sum two-person games that define the unrestricted value of the game as a variable (Gass, 1985) and numerical and statistical problems that utilize linear-programming methods for their solution (Rabinovitz, 1968) etc.

\section{Conclusion}

In this paper we have developed an approach that could be applied to generalized LPs having either free or non-negative variables. By introducing the new rules for entering and leaving variables, the presented approach obviates the need of transforming a given LP involving unrestricted variables into an LP with non-negativity restrictions. Consequently this algorithm saves a lot of computational efforts for larger problems. Computational results, discussed in the end, showed that USM is generally more efficient than SM and works exceptionally well when ratio of number of constraints with number of variables lies near 2 i.e, $m / n \approx 2$.

\section{References:}

1. Chvatal, V. (1983). Linear Programming. United States of America: W.H. Freeman and Company.

2. Dantzig, G. (1963). Linear Programming and Extensions. Princeton University Press.

3. Dantzig, G., \& Thapa, M. (1997). Linear Programming, 1: Introduction. (P. Glynn, Ed.) Springer Verlag.

4. Dines, L. (1918). Systems of inequalities. Annal of Mathematics, 20 (2), 191-198.

5. Gass, S. (1985). Linear Programming Methods and Application. New York: McGraw-Hill.

6. Gass, S. (1985). On the solution of Linear-programming Problems with free variables. Comput. \& Ops. Res., 12, No.3, 265-271. 
7. Goldfarb, D., \& Reid, J. (1977). A practicable steepest edge simplex algorithm. Mathematical Programming, 361-371.

8. Grattan-Guinness, I. (1970). Joseph Fourier's Anticipation of Linear Programming. Operational Research Quarterly, 21(3) , 361-364.

9. Harris, P. (1973). Pivot Selection methods of the Devex LP code. Mathematical Programming, 1-28.

10. Inayatullah, S., Khan, N., Imtiaz, M., \& Khan, F. H. (2010). New Minimum Angle Algorithms for Feasibility and Optimality. Canadian Journal on Computing in Mathematics, Natural Sciences, Engineering \& Medicines., 22-36.

11. Kaluzny, B. (2001). Finite Pivot algoirthms and Feasibility. MS thesis, Faculty of Graduate Studies and Reseach, School of Computer Science, McGill University, Montreal, Quebec, Canada .

12. Motzkin, T. (1952). Contributions to the theory of linear inequalities. RAND Corporation Translation 22.

13. Orchard-Hays, W. (1968). Advanced Linear-Programming Computing Techniques. New york: McGraw-Hill.

14. Pan, P.-Q. (2008). A largest-distance rule for the simplex algorithm. European Journal of Operational Research, 187, No. 2, 393-402.

15. Pan, P.-Q. (2008). Efficient nested pricing in the simplex algorithm. Operations Research Letters, 36, No. 3, 309-313.

16. Pan, P.-Q. (2010). A Fast Simplex Algorithm for Linear Programming. Journal of Computational Mathematics, 28, No.6, 837-847.

17. Rabinovitz, P. (1968, April). Application of linear programming to numerical analysis. SIAM Review, 10.

18. Schechter, M. (1991, June). Unrestricted variables in Linear Programming. Journal of Optimization Theory and Applications, 69.

19. Shamir, R. (1987). The efficiency of simplex method:A survey. Management Science, 301-334.

20. Spivey, W. A., \& Thrall, R. M. (1970). Linear Optimization. New York: Holt, Rinehart \& Winston. 